\title{
Diversidad funcional de murciélagos frugívoros en dos fincas de producción ganadera en Guanacaste, Costa Rica
}

\author{
Fabián Mora-Escobar ${ }^{1}$ (D) María Alejandra Maglianesi ${ }^{2}$ (D) \\ 1. Universidad de Costa Rica, Escuela de Biología, San José, Costa Rica; wfme01@gmail.com \\ 2. Fundación para el Equilibrio entre la Conservación y el Desarrollo (FUNDECODES). Hojancha, Guanacaste, Costa Rica; \\ maria.maglianesicr@gmail.com
}

Recibido 15-III-2021 • Corregido 03-VI-2021 • Aceptado 21-VI-2021

DOI: https://doi.org/10.22458/urj.v13i2.3465

\begin{abstract}
Bat functional diversity in two livestock production farms in Guanacaste, Costa Rica". Introduction: Livestock activities are one of the main causes of the loss and degradation of tropical forests. Consequently, groups of organisms such as bats are affected, impacting the ecosystem services they provide. The analysis of the functional structure of bat assemblages allows to effectively know the characteristics and responses of the species in an ecosystem, thus contributing to the assessment of conservation status of the environments. Objectives: To describe the functional structure of fruit bat assemblages in two livestock farms in Guanacaste, Costa Rica. Methods: Two functional traits related to body size (body mass and forearm length) were measured in fruit bats captured with mist nets in two localities (Nambí and Hojancha). From these traits, the functional diversity of the assemblages was quantified by using the functional richness index, evenness, and functional dispersion, and the weighted mean of these assemblages was calculated. To identify the functional groups of bats, a hierarchical clustering method based on principal component analysis was used, followed by the calculation of Euclidean distances. Results: A total of 191 individuals (14 bat species and two families) were captured. Of the species captured, nine correspond to fruit-eating bats of the subfamilies Stenodermatinae and Carollinae. The bat assemblage of Nambí presented greater functional richness than Hojancha, while the indexes of evenness and functional dispersion were similar in both sites. Conclusion: A higher functional richness of the bat assemblage at Nambí with respect to Hojancha indicates a greater amount of resources exploited by fruit-eating bats. Consequently, bats in this community could be providing greater environmental services compared to Hojancha, which evidences the importance of their conservation for the maintenance of ecological communities in anthropic ecosystems.
\end{abstract}

Keywords: Frugivorous bats, functional dispersion, functional evenness, functional richness, functional traits.
RESUMEN. Introducción: Las actividades ganaderas son una de las principales causas de la pérdida y degradación de los bosques tropicales. Como consecuencia, grupos de organismos como los murciélagos se ven afectados, afectando los servicios ecosistémicos. El análisis de la estructura funcional de los conjuntos de murciélagos permite conocer de forma efectiva las características y respuestas de las especies en un ecosistema, por lo que contribuye a la evaluación del estado de conservación de los ambientes. Objetivos: Describir la estructura funcional de los conjuntos de murciélagos frugívoros en dos fincas ganaderas en Guanacaste, Costa Rica. Métodos: Medimos dos rasgos funcionales relacionados con el tamaño corporal (masa corporal y longitud de antebrazo) en murciélagos frugívoros capturados con redes de niebla en dos localidades (Nambí y Hojancha). Cuantificamos la diversidad funcional de los conjuntos por medio de índices de riqueza funcional, equitatividad, y dispersión funcional, y calculamos la media ponderada de dichos conjuntos. Para identificar los grupos funcionales se utilizó un método de agrupamiento jerárquico basado en el análisis de componentes principales y posteriormente el cálculo de distancias euclidianas. Resultados: Capturamos un total de 191 individuos (14 especies y dos familias). De las especies capturadas, nueve corresponden a frugívoros de las subfamilias Stenodermatinae y Carollinae. El conjunto de murciélagos de Nambí presentó mayor riqueza funcional que el de Hojancha, mientras que los índices de equitatividad y dispersión funcional fueron similares en ambos sitios. Conclusión: Una mayor riqueza funcional del conjunto de murciélagos en Nambí indica una mayor cantidad de recursos explotados por los murciélagos frugívoros. Consecuentemente, los murciélagos en dicha comunidad podrían estar ofreciendo mayores servicios ambientales en comparación con Hojancha, lo cual evidencia la importancia de su conservación para el mantenimiento de comunidades ecológicas en ecosistemas antrópicos.

Palabras clave: Murciélagos frugívoros, dispersión funcional, equitatividad, riqueza funcional, rasgos funcionales. 
La pérdida y degradación de hábitat en los bosques tropicales a causa de la presión antropogénica es uno de los retos más grandes en temas de conservación, siendo la expansión agrícola una de las causas principales (Gardner et al., 2009; Otavo \& Echeverría, 2017). En Costa Rica en las últimas décadas se ha presentado una deforestación significativa, llegando incluso a sobrepasar las proyecciones que se tenían para este fenómeno (Sánchez-Azofeifa et al., 2001). Como ha sucedido en zonas cercanas a áreas protegidas como Tierras Bajas de Tortuguero donde se ha reportado una disminución del $54 \%$ en periodos menores a 10 años (Calvo-Obando, \& OrtizMalavasi, 2012). Gran parte de esta pérdida de hábitat se debe a la expansión de actividades agropecuarias poco planificadas (Silvetti \& Cáceres, 2015).

La disminución de cobertura boscosa se ve reflejada en la pérdida de riqueza y abundancia de especies y las dinámicas de las comunidades (Santos \& Tellería, 2006; Decaëns et al., 2018 ). Grupos de organismos como los murciélagos neotropicales se ven afectados por estos cambios en los ambientes repercutiendo en los servicios ecosistémicos que brindan, tales como la polinización, disminución de artrópodos y dispersión de semillas (Kunz et al., 2011; Castillo-Figueroa, 2020). Las comunidades de murciélagos presentan variaciones con respecto al grado de tolerancia a alteraciones en sus hábitats. Algunas especies como Vampirum spectrum son dependientes de bosques maduros (Vargas-Espinoza et al., 2004), mientras que algunas del género Artibeus, Sturnira y Molossus se los puede encontrar en ecosistemas antrópicos donde se alimentan, por ejemplo, de cultivos de diversos tipos (Kalda et al., 2015; Heim, et al., 2016; Huang et al., 2019) o incluso zonas urbanizadas (Coleman, \& Barclay, 2012; Rodríguez-Aguilar et al., 2017).

Conocer la tolerancia a los ambientes perturbados de diferentes especies de murciélagos brinda la posibilidad de monitorear la salud de una variedad de ecosistemas y la respuesta de las especies a los cambios percibidos en el ambiente (Santos \& Tellería, 2006) brindando información de las comunidades de plantas e insectos de las cuales se alimentan (Jones et al., 2009). Gremios tróficos como los murciélagos frugívoros constituyen además un excelente indicador de los recursos disponibles en el ambiente en el que se desarrollan debido a sus necesidades de hábitat para forrajeo y alimentación. Además, este gremio de murciélagos puede orientar algunas posibles soluciones con relación a la regeneración de ambientes degradados por el papel que desempeñan en la dispersión de semillas de plantas pioneras (Charles-Dominique, 1986; Muscarella \& Fleming, 2007; Cely-Gómez, \& Castillo-Figueroa, 2019). Así, en áreas agropecuarias, se pueden observar especies típicas de ambientes con un mayor grado de afectación y analizando la composición de las comunidades es posible describir el estado de tales ambientes (Santos \& Tellería, 2006).

La estructura de las comunidades ecológicas se puede describir por medio de los rasgos funcionales de las especies, definidos como características ya sean morfológicas, fisiológicas, conductuales o incluso reproductivas de los individuos, que influyen significativamente en su desempeño en el ambiente donde se encuentran y afectan niveles organizacionales mayores (Violle et al., 2007; Mokany et al., 2008). En nuestro estudio nos enfocamos a analizar rasgos morfológicos exclusivamente. La composición de rasgos de una comunidad puede ser examinada por medio de dos componentes: (a) el valor promedio de los rasgos de las especies, ponderados por sus abundancias relativas y (b) medidas multivariadas de diversidad funcional (Dias et al., 2013). La diversidad funcional describe el rango, distribución y abundancia de valores característicos de las especies en una comunidad dada (Tilman et al., 1997).

A partir de diversos índices de diversidad funcional es posible distinguir y agrupar los organismos con relación al medio donde se encuentran y las interacciones que presentan con otros organismos (Petchey \& Gaston, 2006). A su vez, los rasgos funcionales y la diversidad funcional de las comunidades permiten conocer de forma efectiva las propiedades y respuestas de las especies en un ecosistema a diferencia de la diversidad taxonómica tradicionalmente empleada (Mokany et al., 2008). El presente estudio se llevó a cabo en dos fincas ganaderas en el noroeste de Costa Rica 
donde existen diversos elementos en el paisaje que ofrecen hábitat a las especies de murciélagos. Sin embargo, se cuenta con poca información sobre la estructura funcional de los conjuntos de murciélagos en esta región y el rol que los bosques remanentes en fincas ganaderas podrían desempeñar en la conservación de especies de murciélagos. El objetivo de este estudio es describir la diversidad funcional de dos conjuntos de murciélagos frugívoros en fincas ganaderas que permita conocer el estado actual de los hábitats donde se encuentran y orienten acciones de conservación.

\section{MATERIALES Y MÉTODOS}

Área de estudio: El estudio se realizó en el noroeste de Costa Rica en la parte central de la Península de Nicoya en dos localidades: Nambí $\left(10^{\circ} 12^{\prime} 19^{\prime \prime} \mathrm{N}, 85^{\circ} 30^{\prime} 15^{\prime \prime} \mathrm{W}\right)$ y Hojancha $\left(10^{\circ} 04^{\prime} 21^{\prime \prime} \mathrm{N}\right.$, $85^{\circ} 25^{\prime} 31^{\prime \prime O}$ ) (Fig. 1.). Esta área es parte de la provincia de Guanacaste y corresponde a la zona de vida del Bosque Húmedo Tropical (Holdridge, 1967). En esta zona, la altitud varía de 130 a 385 msnm, mientras que la temperatura media anual es de $26,8^{\circ} \mathrm{C}$ en promedio y la precipitación media anual es de $2400 \mathrm{~mm}$. La estación seca dura de enero a abril, y los meses más lluviosos son julio, octubre y noviembre.

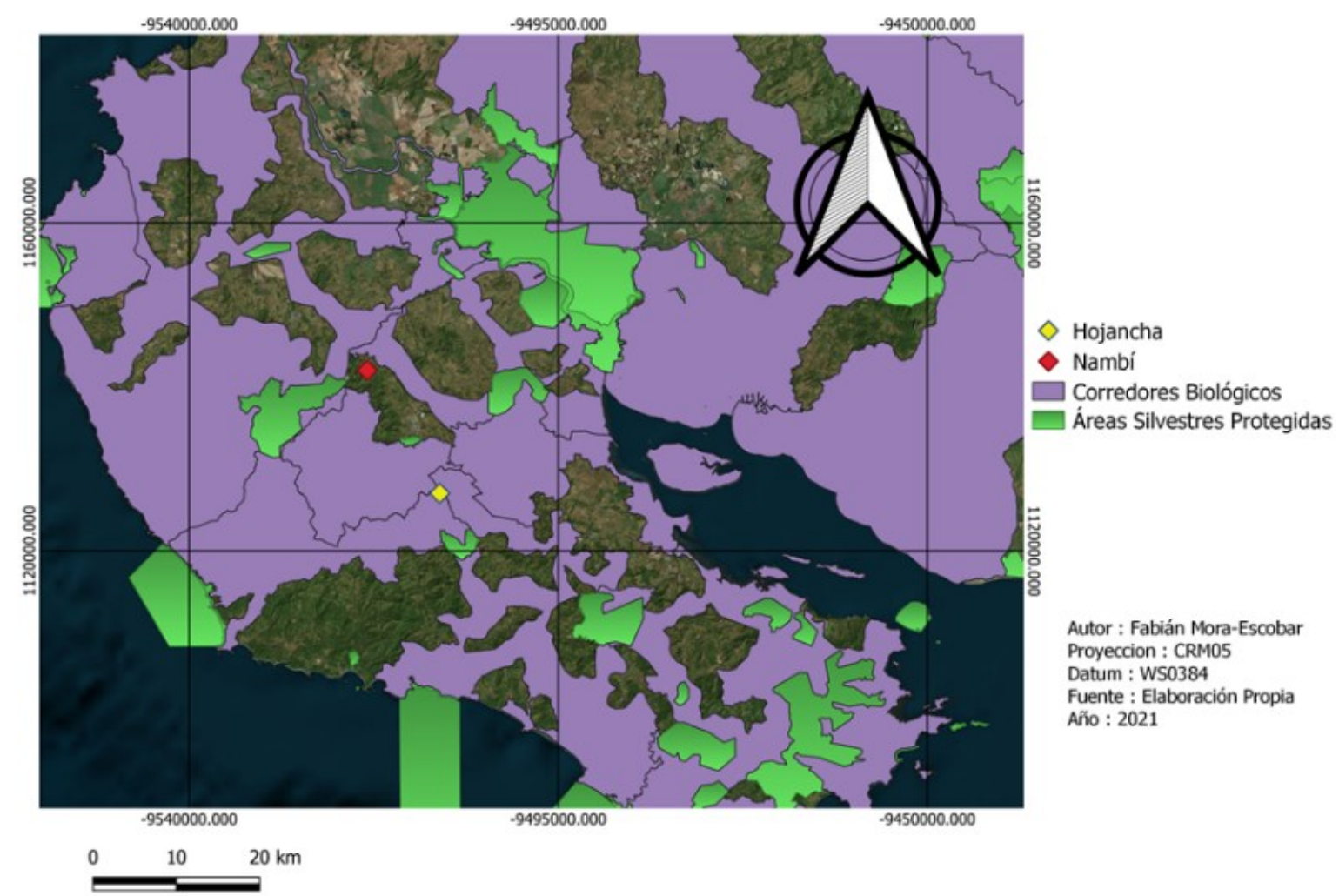

Fig. 1. Ubicación de los sitios de muestreo y áreas con algún grado de conservación en la Península de Nicoya, provincia de Guanacaste, Costa Rica

La recolección de datos de campo se realizó en dos fincas con características del paisaje distintas (Fig. 1). Nambí presenta cuerpos de agua dentro del área de muestreo y se ubica a $2.7 \mathrm{~km}$ con respecto al Parque Nacional Diría, siendo esta el área protegida más cercana, así mismo el área de muestreo no presenta ninguna categoría de protección (Fig. 2). Con respecto a Hojancha, no se tienen ríos cercanos al área de muestreo, el área protegida más cercana es la Zona Protectora 
Montealto que se ubica a 4,3 km de distancia y es parte del Programa Nacional de Corredores Biológicos (Fig. 3). Entre el área de muestreo y dicha zona protectora, se tiene un área mayormente urbanizada.

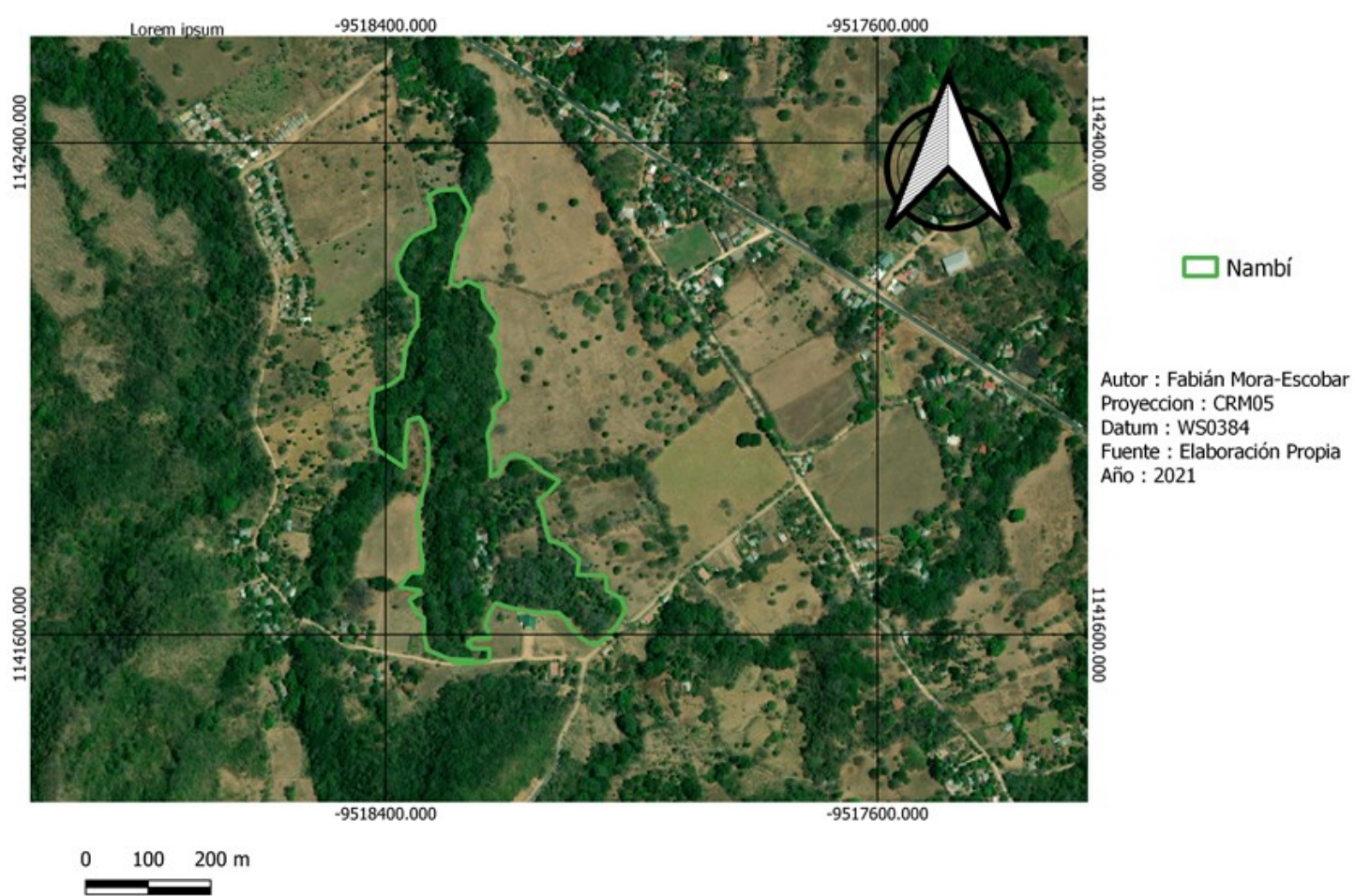

Fig. 2. Área de muestreo en la localidad de Nambí, Guanacaste, Costa Rica

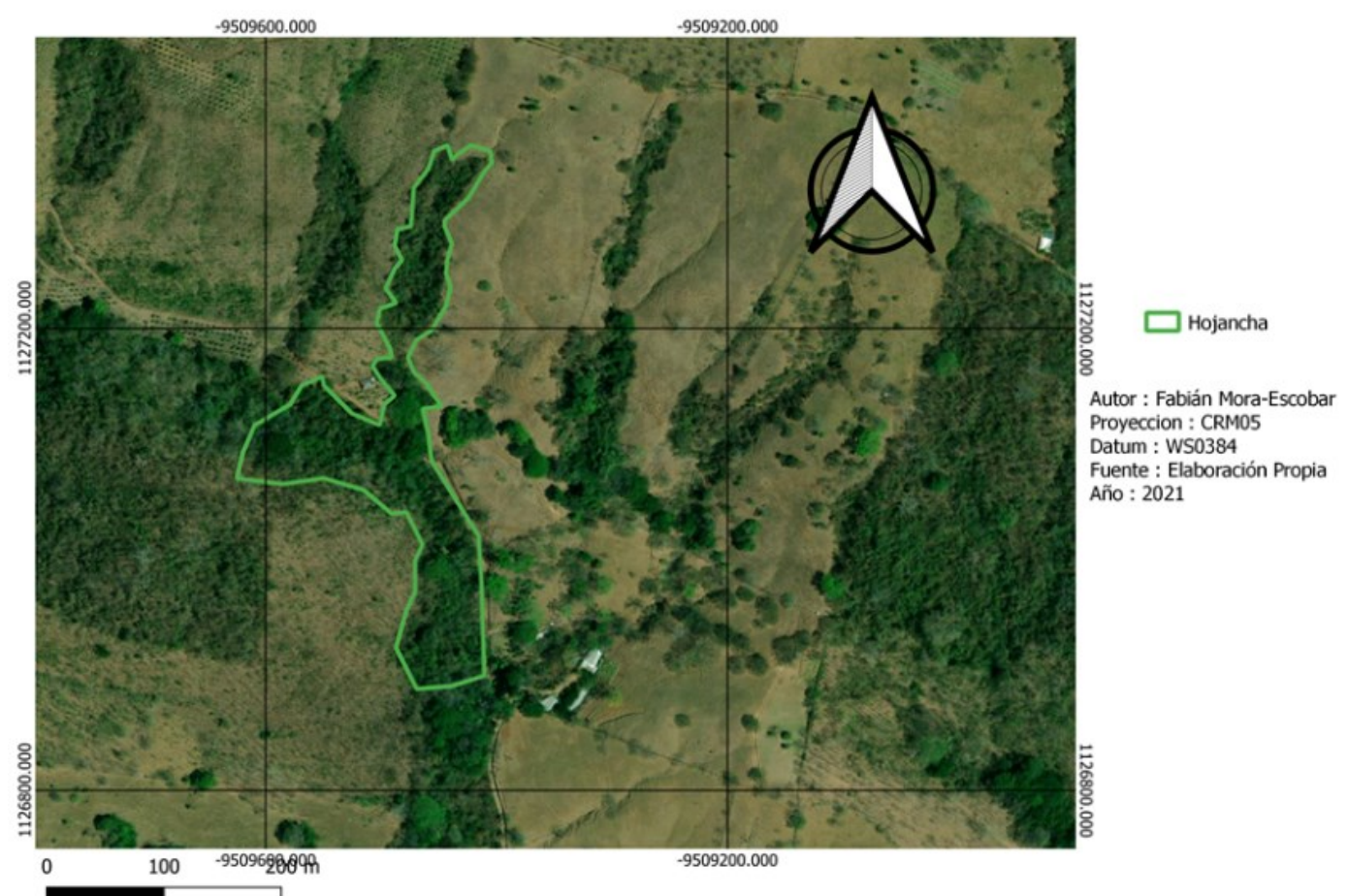

Fig. 3. Área de muestreo en la localidad de Hojancha, Guanacaste, Costa Rica. 
Recolección de datos: El trabajo de campo se realizó de enero a agosto de 2019 durante dos noches por sitio tanto en estación seca como lluviosa para captar la variación que puede darse en la composición de murciélagos entre diferentes estaciones dentro de un mismo año. Para obtener medidas morfológicas, los murciélagos fueron capturados utilizando cinco redes de niebla estándares $(12 \times 3 \mathrm{~m}$ ) colocadas de forma aleatoria en bosque secundario en cada finca. La captura se realizó desde las 6:00 pm hasta las 2:00 am con un esfuerzo de muestreo de 40 horas-red por noche (Moreno \& Halffter, 2000). Los muestreos no se realizaron en días de luna llena para evitar que pudiera darse una baja tasa de captura de murciélagos. Es importante considerar que al usar redes de niebla sólo es posible capturar especies de murciélagos cuya actividad corresponda al sotobosque, por lo tanto, este estudio se enfocó a las especies frugívoras en dicho estrato de vegetación. Todos los murciélagos capturados fueron identificados a nivel de especie (Timm et al., 1999). Para cada individuo se determinó la edad, sexo y estadio reproductivo, y entre los rasgos morfológicos se midieron la longitud del antebrazo y la masa corporal. Cada individuo fue marcado temporalmente utilizando pintura acrílica en las garras a los fines de diferenciarlo cuando se tratara de una recaptura. La masa corporal está relacionada con la cantidad y calidad de alimentos obtenidos, lo cual influye en la supervivencia de los murciélagos (Castillo-Figueroa \& Pérez-Torres, 2021). Mientras que la longitud del antebrazo se encuentra directamente relacionada con la capacidad de vuelo y la manipulación de frutos de distintos tamaños (Muñoz-Romo \& Herrera, 2010), por lo que es de gran importancia en procesos de dispersión de semillas. A partir de las capturas se determinó la abundancia relativa de cada especie de murciélago, para lo cual se descartaron las recapturas para evitar una sobre estimación en la abundancia de las especies.

Análisis de datos: Para analizar diferencias en las medidas morfométricas entre las diferentes especies de murciélagos, se realizó una prueba de Análisis de Varianza incluyendo la masa o la longitud del antebrazo como variable respuesta y las especies de murciélagos como variable explicativa (Tabla 1). Con el fin de determinar la estructura funcional de los conjuntos de murciélagos frugívoros, se calcularon dos componentes de la composición de rasgos de las comunidades: (a) la media ponderada de la comunidad (CWM, por sus siglas en inglés) y (b) el grado en que los valores de los rasgos difieren entre las especies de murciélagos, cuantificado por tres medidas de diversidad funcional. CWM se cuantificó como el valor medio de cada rasgo para todas las especies de murciélagos presentes en la comunidad, ponderado por sus abundancias relativas obtenidas a partir de las capturas con redes de niebla en cada finca estudiada (Garnier et al., 2004; Lavorel et al., 2008). Para calcular la diversidad funcional, las especies de murciélagos se proyectaron en un espacio de rasgos basado en distancias euclidianas por pares, calculadas a partir de los rasgos funcionales mediante el análisis de coordenadas principales (Villéger et al., 2008).

Se utilizaron tres índices de diversidad funcional: riqueza funcional (FRic), dispersión funcional (FDis) y equitatividad funcional (FEve), los cuales describen de manera complementaria diferentes atributos de la diversidad funcional del conjunto de especies (Mason et al., 2005, Villéger et al., 2008). FRic brinda información sobre los recursos o espacios ocupados por la comunidad con respecto a los rasgos funcionales estudiados (Cornwell et al., 2006). Mientras que FDis indica el nivel de heterogeneidad de los distintos rasgos funcionales estudiados (Laliberté \& Legendre, 2010) y FEve por su parte proporciona información sobre la uniformidad en la distribución de las abundancias de especies en su nicho (Mason et al., 2005).

Para identificar los grupos funcionales de murciélagos, se utilizó el método de agrupamiento jerárquico de Ward (Ward, 1963), el cual se basa en un análisis de componentes principales y posteriormente el cálculo de distancias euclidianas, a partir de las cuales se generó un dendrograma. Todos los análisis se realizaron con el lenguaje de programación R Core Team (2020). Para calcular las métricas de diversidad funcional y realizar el análisis de agrupamiento se utilizó la función de 
Índices de Diversidad Funcional Basados en la Distancia (dbFD) en el paquete "FD" (Laliberté et al., 2015).

\section{RESULTADOS}

Se capturaron un total de 191 individuos pertenecientes a 14 especies de murciélagos y dos familias (41 en Hojancha y 150 en Nambí). La familia Phyllostomidae fue la más representada y cuatro subfamilias presentes en ambos sitios (Tabla 1). De las especies capturadas, nueve corresponden a frugívoros de las subfamilias Stenodermatinae y Carollinae (Fig. 4). Artibeus jamaicensis fue la especie con mayor abundancia, seguida por Artibeus watsoni y Artibeus lituratus (Fig. 4). Se identificaron cuatro grupos funcionales de murciélagos en donde A. lituratus y $A$. jamaicensis conformaron el grupo que mostró una mayor distancia en el dendrograma con respecto a las otras especies de murciélagos frugívoros debido a un mayor valor en los rasgos asociados con el tamaño corporal (masa y longitud de antebrazo), (Fig. 5). Se encontraron diferencias tanto en las medidas de masa corporal $(F=165, p<0,001, n)$ como de longitud de antebrazo $(F=526,6, P<$ 0,001 ) entre las especies de murciélagos frugívoros. En general, murciélagos como $A$. lituratus y $A$. jamaicensis presentaron mayor masa corporal y longitud de antebrazo.

TABLA 1

Especies de murciélagos frugívoros registrados en dos fincas ganaderas de la región central de la Península de Nicoya, Guanacaste Costa Rica. Los nombres y clasificación taxonómica siguen la descripción de York et al. (2019).

\begin{tabular}{lllc}
\hline \multicolumn{1}{c}{ Familia } & \multicolumn{1}{c}{ Subfamilia } & \multicolumn{1}{c}{ Especie } & Nombre común \\
\hline Phyllostomidae & Glossophaginae & Glossophaga leachii & Murciélago de Leach \\
Phyllostomidae & Glossophaginae & Glossophaga soricina & Murciélago musaraña \\
Phyllostomidae & Carollinae & Carollia perspicillata & Murciélago candelero \\
Phyllostomidae & Carollinae & Carollia subrufa & Carolia parda \\
Phyllostomidae & Stenodermatinae & Artibeus jamaicensis & Artibeo jamaiquino \\
Phyllostomidae & Stenodermatinae & Artibeus lituratus & Artibeo correcto \\
Phyllostomidae & Stenodermatinae & Artibeus phaeotis & Dermanura pardo \\
Phyllostomidae & Stenodermatinae & Artibeus watsoni & Dermanura de Watson \\
Phyllostomidae & Stenodermatinae & Platyrrhinus heleri & Murciélago de Heller \\
Phyllostomidae & tenodermatinae & Sturnira parvidens & Murciélago de hombros \\
Phyllostomidae & Stenodermatinae & Uroderma convexum & Murciélago bilobulado \\
\hline
\end{tabular}

La diversidad taxonómica de murciélagos frugívoros en los dos sitos estudiados resultó muy similar variando solamente en dos especies (Fig. 4). Para las medias ponderadas a nivel de comunidad de valores de rasgos (CWM) correspondiente a la longitud de antebrazo, se encontró que fue ligeramente mayor para Hojancha con respecto a Nambí (Tabla 2). Con base a los rasgos de masa corporal y longitud de antebrazo en gremios de murciélagos frugívoros, se encontró que la diversidad funcional en términos de riqueza funcional (FRic) del conjunto de murciélagos frugívoros en Nambí fue mayor que para Hojancha (Tabla 2). En el caso de la dispersión funcional (FDis) y equitatividad (FEve), se encontraron valores cercanos para ambos sitios de estudio (Tabla 2). 
TABLA 2

Índices de diversidad funcional y media ponderada a nivel de comunidad en conjuntos de murciélagos frugívoros en dos fincas ganaderas en la Península de Nicoya, Guanacaste, Costa Rica.

\begin{tabular}{cccccc}
\hline \multirow{2}{*}{ Sitio } & \multicolumn{2}{c}{ Índice de diversidad funcional } & \multicolumn{2}{c}{ Media ponderada } \\
\cline { 2 - 6 } & Riqueza & Dispersión & Equitatividad & Masa & Antebrazo \\
\hline Hojancha & 0,48 & 1,25 & 0,49 & 22,26 & 46,71 \\
Nambí & 0,78 & 1,07 & 0,44 & 22,59 & 44,90 \\
\hline
\end{tabular}

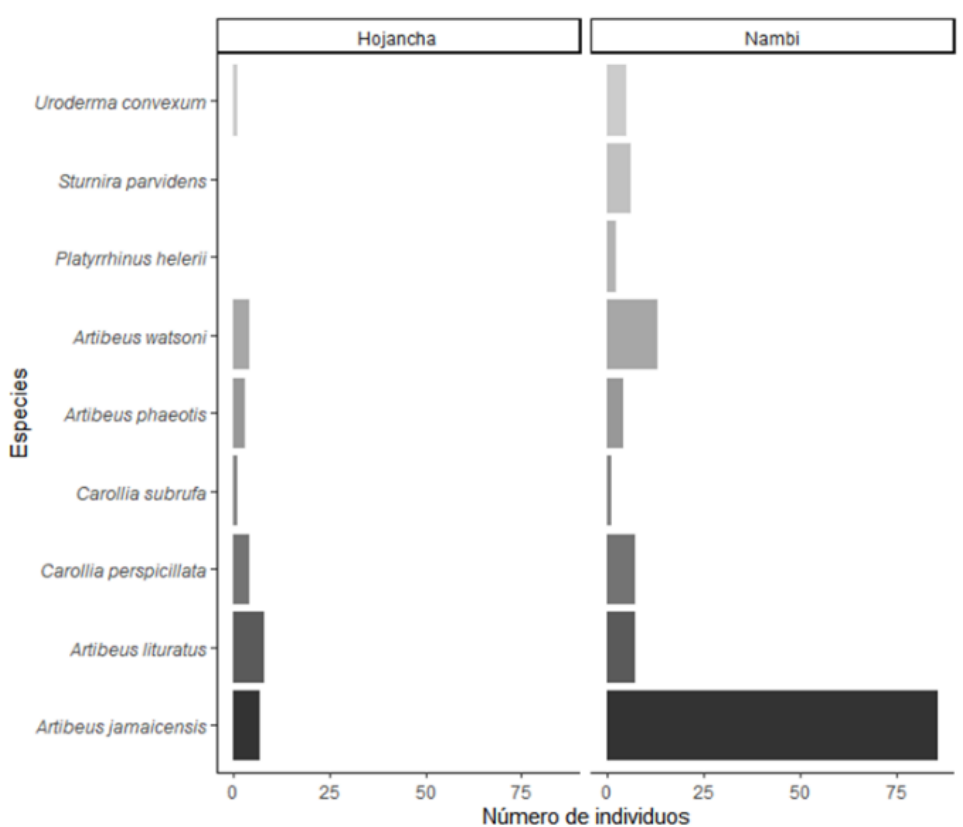

Fig. 4. Número de individuos de especies de murciélagos frugívoros (Phyllostomidae) capturados en dos localidades en la Península de Nicoya, Guanacaste, Costa Rica.

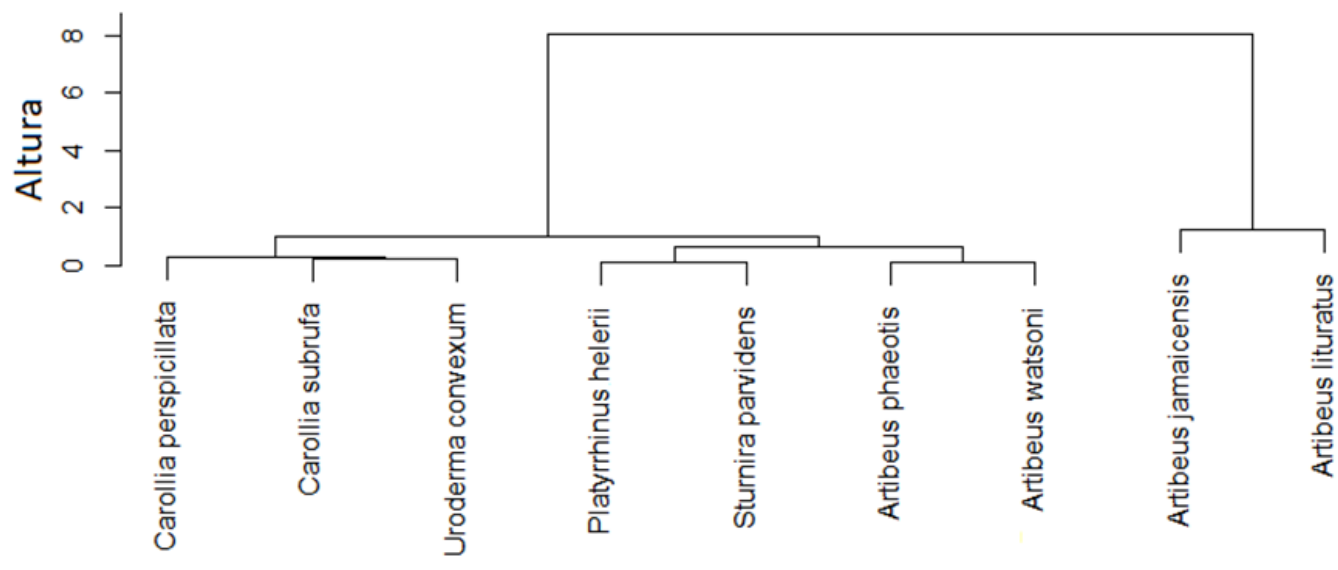

\section{Distancia}

Fig. 5. Dendrograma resultante del análisis de agrupamiento jerárquico de especies de murciélagos frugívoros (Phyllostomidae) basado en dos rasgos funcionales (masa corporal y longitud del antebrazo) en dos localidades en la Península de Nicoya, Costa Rica. 


\section{DISCUSIÓN}

La riqueza taxonómica de murciélagos frugívoros en este estudio fue mayor que la reportada para zonas cercanas a áreas que presentan un grado relativamente alto de urbanismo (Arias-Aguilar et al., 2015). Estos resultados pueden deberse a la cercanía de ciertos tipos de cobertura arbórea que brindan una mayor disponibilidad de recursos para los murciélagos, tales como bosques de galería y cercas vivas, entre otros. Las especies encontradas en ambos sitios estudiados corresponden a especies comúnmente generalistas y habituales de la zona (York, et al., 2019), o con requerimientos de alimentación y refugio poco exigentes (Saldaña-Vázquez et al., 2010; Ferreira, et al., 2017; Garbino \& Tavares, 2018). Tal es el caso de Artibeus jamaicensis y especies del género Carollia (Rubí-Chacón et al., 2018).

Tomando en cuenta que FRic de un conjunto de especies es una medida independiente de la abundancia de individuos (Villéger et al., 2010), la diferencia en esta métrica entre los sitios estudiados podría deberse a una variación en el número de especies y en los rasgos funcionales considerados. Así, las dos especies que fueron encontradas únicamente en el sitio con mayor FRic (Sturnira parvidens y Platyrrhinus helleri) constituyen un grupo bien diferenciado dentro del conjunto completo de murciélagos frugívoros debido a que sus medidas morfológicas difieren en conjunto de forma considerable con relación a las otras especies, estas especies se han visto agrupadas de una forma similar en otros estudios sobre diversidad funcional en fincas ganaderas (Castillo-Figueroa \& Pérez-Torres, 2018)

Con respecto a los murciélagos frugívoros en el neotrópico encontramos grupos como la subfamilia Carollinae, la cual cumple un papel de dispersión de plantas como las del género Pipper, cuyos frutos son de un tamaño reducido (Fleming \& Heithaus, 1986; Ferreira, et al., 2017). Mientras que los murciélagos de la subfamilia Stenodermatinae tienen una capacidad de dispersión de semillas de frutos de tamaños más variables llegando incluso a manipular frutos de especies de Ficus y Spondias (Passos \& Passamani, 2003; Saldaña-Vázquez et at., 2010; Garbino, \& Tavares, 2018). Los murciélagos de estos dos grupos fueron separados claramente según sus rasgos funcionales en los sitios estudiados. Una menor FRic en Hojancha con respecto a Nambí sugiere la posibilidad de que haya recursos potencialmente disponibles que no están siendo explotados por los murciélagos en dicha localidad (Mason et at., 2005). Esta subutilización del nicho alimenticio de los murciélagos podría consecuentemente resultar en una reducción en los servicios ambientales que brindan. Contrariamente, una mayor cantidad de recursos explotados por los murciélagos frugívoros dispersores de semillas podría beneficiar a las plantas en términos reproductivos, contribuyendo a una mayor riqueza vegetal en las comunidades (Saldaña-Vázquez et al., 2010; Ferreira et al., 2017; Garbino, \& Tavares, 2018). Es importante realizar estudios a futuro que incluyan otras medidas morfométricas a las consideradas en nuestro estudio y el grado de especialización alimenticia a los fines de comprender mejor la relación entre la estructura funcional de los conjuntos de murciélagos y el uso de los hábitats.

Para los valores de FDis se encontró que las especies dominantes y, por lo tanto, las medidas de los rasgos fueron muy similares entre ambos sitios, lo cual sugiere una utilización similar de los recursos por los murciélagos en ambas comunidades (Mason et al., 2005; Mason et al., 2013). Por otra parte, la medida de FEve depende en parte de la distribución de abundancias de las especies, la cual resultó ser similar en ambos sitios de estudio, con una variación importante sólo en dos especies (Villéger et al., 2010). De igual manera, para CWM se obtuvieron valores muy similares para ambos sitios, lo cual puede deberse a que esta métrica se basa en la abundancia relativa de las especies con respecto al valor del rasgo. En ambos conjuntos la mayoría de las especies tuvieron una tasa de captura de individuos similar, explicando así la similitud entre dichos conjuntos de murciélagos frugívoros para los dos rasgos estudiados (Bellwood et al., 2006). 
En conclusión, ambos sitios muestran un conjunto de murciélagos frugívoros similar excepto en términos de FRic, lo cual sugiere que Nambí presenta una mayor cantidad de recursos utilizados por la comunidad. Consecuentemente, los murciélagos en esta comunidad podrían ser más efectivos en cuanto a los servicios ambientales de dispersión de semillas que brindan en comparación con Hojancha, especialmente en el transporte de semillas grandes como las de Ficus y Spondias, además de las especies pioneras habituales como Pipper. Esto evidencia la necesidad de conservar las especies de murciélagos que contribuyen con el mantenimiento de las comunidades ecológicas. Se espera que estas métricas sirvan como base para orientar proyectos de regeneración de bosques en el área y poder ser comparados en el futuro. Así, los valores de FRic, FEve y FDiv podrían ser mayores con respecto a los ya calculados en ambientes donde la vegetación se haya regenerado, lo cual se traduce en conjuntos más complejos característicos de áreas con mayor grado de conservación (Mason et al., 2005; Villéger et al., 2010).

\section{AGRADECIMIENTOS}

Agradecemos al financiamiento otorgado por el I Canje de Deuda por Naturaleza EE.UU.C.R. gestionado por la Asociación Costa Rica por Siempre. Queremos agradecer a Eduardo Artavia, a los asistentes de campo y voluntarios de las comunidades locales quienes contribuyeron con la recolección de datos. También al Sistema Nacional de Áreas de Conservación (SINAC) por otorgar el permiso de investigación para realizar muestreos de campo. Además, agradecemos a los propietarios Javier Zúñiga Díaz y Alexis Zúñiga por la colaboración para desarrollar el proyecto en sus fincas. Por último, agradecemos a Jeremy Klank, por la asesoría y ayuda en el análisis estadístico de rasgos funcionales.

\section{ÉTICA, CONFLICTO DE INTERESES Y DECLARACIÓN DE FINANCIAMIENTO}

Los autores declaran haber cumplido con todos los requisitos éticos y legales pertinentes, tanto durante el estudio como en el manuscrito; que no hay conflictos de interés de ningún tipo, y que todas las fuentes financieras se detallan plena y claramente en la sección de agradecimientos. Asimismo, están de acuerdo con la versión editada final del documento. El respectivo documento legal firmado se encuentra en los archivos de la revista.

La declaración de la contribución de cada autor al manuscrito es la siguiente: F.M.E.: estudiante, realizó los análisis de datos y elaboró el artículo. M.A.M.: investigadora, coordinó la colecta de datos de campo y contribuyó con la elaboración del artículo. Ambos propusieron las ideas del artículo. 


\section{REFERENCIAS}

Arias-Aguilar, A., Chacón-Madrigal, E., \& Rodríguez-Herrera, B. (2015). El uso de los parques urbanos con vegetación por murciélagos insectívoros en San José, Costa Rica. Mastozoología neotropical, 22(2), 229-237.

Bellwood, D. R., Wainwright, P. C., Fulton, C. J., \& Hoey, A. S. (2006). Functional versatility supports coral reef biodiversity. Proceedings of the Royal Society, 273(1582), 101-107. https://doi.org/10.1098/rspb.2005.3276

Calvo-Obando, A., \& Ortiz-Malavasi, E. (2012). Fragmentación de la cobertura forestal en Costa Rica durante los períodos 1997-2000 y 2000-2005. Revista Forestal Mesoamericana Kurú, 9(22), 10-21 https://doi.org/10.18845/rfmk.v9i22.359

Castillo-Figueroa, D. (2020). Why bats matters: a critical assessment of bat-mediated ecological processes in the Neotropics. European Journal of Ecology, 6(1), 77-101. https://doi.org/10.17161/eurojecol.v6i1.13824

Castillo-Figueroa, D., \& Pérez-Torres, J. (2018). Respuestas funcionales de murciélagos asociados a fragmentos de bosque seco tropical en Córdoba (Colombia): implicaciones del tipo de manejo en sistemas de ganadería extensiva. Revista Biodiversidad Neotropical, 8(3), 197-211.

Castillo-Figueroa, D., \& Pérez-Torres, J. (2021). On the development of a trait-based approach for studying Neotropical bats. Papéis Avulsos de Zoologia, 61, e20216124. https://doi.org/10.11606/1807-0205/2021.61.24

Cely-Gómez, M. A., \& Castillo-Figueroa, D. (2019). Diet of dominant frugivorous bat species in an oil palm landscape from Colombian Llanos: implications for forest conservation and recovery. Therya, 10(2), 149-153. https://doi.org/10.12933/therya-19-682

Charles-Dominique, P. (1986). Inter-relations between frugivorous vertebrates and pioneer plants: Cecropia, birds and bats in French Guyana. En A. Estrada \& T. Fleming (Eds.). Frugivores and seed dispersal (pp. 119-135).Springer. https://doi.org/10.1007/978-94-009-4812-9 12

Coleman, J. L., \& Barclay, R. M. (2012). Urbanization and the abundance and diversity of Prairie bats. Urban Ecosystems, 15(1), 87-102. https://doi.org/10.1007/s11252-011-0181-8

Cornwell, W. K., Schwilk, W. K., Ackerly, D. D. (2006). A trait-based test for habitat filtering: convex hull volume. Ecology, 87, 1465-1471. https://doi.org/10.1890/0012-9658(2006)87[1465:ATTFHF]2.0.CO;2

Dias, A. T. C., Berg, M. P., de Bello, F., Van Oosten, A. R., Bílá, K., \& Moretti, M. (2013). An experimental framework to identify community functional components driving ecosystem processes and services delivery. Journal of Ecology, 101(1), 29-37. https://doi.org/10.1111/1365-2745.12024

Ferreira, D. F., Rocha, R., López-Baucells, A., Farneda, F. Z., Carreiras, J. M., Palmeirim, J. M., \& Meyer, C. F. (2017). Seasonmodulated responses of Neotropical bats to forest fragmentation. Ecology and Evolution, 7(11), 4059-4071. https://doi.org/10.1002/ece3.3005

Fleming, T. H., \& Heithaus, E. R. (1986). Seasonal foraging behavior of the frugivorous bat Carollia perspicillata. Journal of Mammalogy, 67(4), 660-671. https://doi.org/10.2307/1381127

Garbino, G. S., \& Tavares, V. (2018). Roosting ecology of Stenodermatinae bats (Phyllostomidae): evolution of foliage roosting and correlated phenotypes. Mammal Review, 48(2), 75-89. https://doi.org/10.1111/mam.12114

Gardner, T. A., Barlow, J., Chazdon, R., Ewers, R. M., Harvey, C. A., Peres, C. A., \& Sodhi, N. S. (2009). Prospects for tropical forest biodiversity in a human-modified world. Ecology letters, 12(6), 561-582. https://doi.org/10.1111/j.1461$\underline{0248.2009 .01294 . x}$

Garnier, E., Cortez, J., Bille's, G., Navas, M. L., Roumet, C., Debussche, M., Toussaint, J. P. (2004). Plant functional markers capture ecosystem properties during secondary succession. Ecology, 85(9), 2630-2637. https://doi.org/10.1890/03-0799 
Heim, O., Schröder, A., Eccard, J., Jung, K., \& Voigt, C. C. (2016). Seasonal activity patterns of European bats above intensively used farmland. Agriculture, Ecosystems \& Environment, 233, 130-139. https://doi.org/10.1016/j.agee.2016.09.002

Holdridge, L. R. (1967). Life zone ecology. San José, Costa Rica: Tropical Science Center,

Huang, J. C. C., Rustiati, E. L., Nusalawo, M., \& Kingston, T. (2019). Echolocation and roosting ecology determine sensitivity of forest-dependent bats to coffee agriculture. Biotropica, 51(5), 757-768. https://doi.org/10.1111/btp.12694

Jones, G., Jacobs, D. S., Kunz, T. H., Willig, M. R., \& Racey, P. A. (2009). Carpe noctem: the importance of bats as bioindicators. Endangered species research, 8(1-2), 93-115. https://doi.org/10.3354/esr00182

Kalda, O., Kalda, R., \& Liira, J. (2015). Multi-scale ecology of insectivorous bats in agricultural landscapes. Agriculture, Ecosystems \& Environment, 199, 105-113. https://doi.org/10.1016/j.agee.2014.08.028

Kunz, T. H., Torrez, E. B., Bauer, D., Lobova, T., \& Fleming, T. H. (2011). Ecosystem services provided by bats. Annals of the New York Academy of Sciences, 1223, 1-38. https://doi.org/10.1111/i.1749-6632.2011.06004.x

Laliberté, E., \& Legendre P. (2010). A distance-based framework for measuring functional diversity from multiple traits. Ecology, 91, 299-305. https://doi.org/10.1890/08-2244.1

Laliberté, E., Legendre, P., \& Shipley, B. (2015). Package FD. Measuring functional diversity (FD) from multiple traits, and other tools for functional ecology v 1.0-12. https://cran.r-project.org/web/packages/FD/FD.pdf

Lavorel, S., Grigulis, K., McIntyre, S., Williams, N., Garden, D., Dorrough, J., Berman, S., Quétier, F., Thébault, A., \& Bonis, A. (2008). Assessing functional diversity in the field - methodology matters! Functional Ecology, 22(1), 134-147.

Mason, N., de Bello, F., Mouillot, D., Pavoine, S., \& Dray, S. (2013). A guide for using functional diversity indices to reveal changes in assembly processes along ecological gradients. Journal of Vegetation Science, 24(5), 794-806. https://doi.org/10.1111/jvs.12013

Mason, N., Mouillot, D., Lee, W., \& Wilson, B. (2005). Functional richness, functional evenness and functional divergence: the primary components of functional diversity. Oikos, 111(1), 112-118. https://doi.org/10.1111/j.0030$\underline{1299.2005 .13886 . x}$

Mokany, K., Ash, J., \& Roxburgh, S. (2008). Functional identity is more important than diversity in influencing ecosystem processes in a temperate native grassland. Journal of Ecology, 96(5), 884-893. https://doi.org/10.1111/i.1365$\underline{2745.2008 .01395 . x}$

Moreno, C. E., \& Halffter, G. (2000). Assessing the completeness of bat biodiversity inventories using species accumulation curves. Journal of Applied Ecology, 37, 149-158. https://doi.org/10.1046/i.1365-2664.2000.00483.x

Muñoz-Romo, M., \& Herrera, E. A. (2010). Observaciones sobre la alimentación del murciélago frugívoro mayor Artibeus lituratus (Chiroptera: Phyllostomidae) en Venezuela. Revista Mexicana de Mastozoología (Nueva Época), 14(1), 51-58. https://doi.org/10.22201/ie.20074484e.2010.14.1.27

Muscarella, R., \& Fleming, T. H. (2007). The role of frugivorous bats in tropical forest succession. Biological reviews, 82(4), 573-590. https://doi.org/10.1111/i.1469-185X.2007.00026.x

Otavo, S., \& Echeverria, C. (2017). Progressive fragmentation and loss of natural forests habitat in one of the global biodiversity hotspot. Revista Mexicana de Biodiversidad, 88(4), 924-935. https://doi.org/10.1016/j.rmb.2017.10.041

Passos, J. G., \& Passamani, M. (2003). Artibeus lituratus (Chiroptera, Phyllostomidae): biologia e dispersão de sementes no Parque do Museu de Biologia Prof. Mello Leitão, Santa Teresa (ES). Natureza on line, 1(1), 1-6.

Petchey, O. L., \& Gaston, K. J. (2006). Functional diversity: back to basics and looking forward. Ecology letters, 9(6), 741758. https://doi.org/10.1111/j.1461-0248.2006.00924.x 
R Core Team (2020). R Foundation for Statistical Computing. https://www.R-project.org/

Rodríguez-Aguilar, G., Orozco-Lugo, C. L., Vleut, I., \& Vazquez, L. B. (2017). Influence of urbanization on the occurrence and activity of aerial insectivorous bats. Urban ecosystems, 20(2), 477-488. https://doi.org/10.1007/s11252$\underline{016-0608-3}$

Rubí-Chacón, R., Urbina-Villalobos, A., Herrero, M., \& Dolz, G. (2018). Murciélagos generalistas de Costa Rica: descripción macroambiental de sitios de captura, características de hospedero y caracterización molecular de Leishmania spp. y Trypanosoma spp. Ciencias Veterinarias, 36(3), 39. https://doi.org/10.15359/rcv.36-3.28

Saldaña-Vázquez, R. A., Sosa, V. J., Hernández-Montero, J. R., \& López-Barrera, F. (2010). Abundance responses of frugivorous bats (Stenodermatinae) to coffee cultivation and selective logging practices in mountainous central Veracruz, Mexico. Biodiversity and Conservation, 19(7), 2111-2124. https://doi.org/10.1007/s10531-010-9829$\underline{6}$

Sánchez-Azofeifa, G. A., Harriss, R. C., \& Skole, D. L. (2001). Deforestation in Costa Rica: a quantitative analysis using remote sensing imagery 1. Biotropica, 33(3), 378-384. https://doi.org/10.1111/j.1744-7429.2001.tb00192.x

Santos, T., \& Tellería, J. L. (2006). Pérdida y fragmentación del hábitat: efecto sobre la conservación de las especies. Revista Ecosistemas, 15(2).

Silvetti, F., \& Cáceres, D. M. (2015). La expansión de monocultivos de exportación en Argentina y Costa Rica: Conflictos socioambientales y lucha campesina por la justicia ambiental. Mundo Agrario, 16(32).

Tilman, D., Knops, J., Wedin, D., Reich, P., Ritchie, M., \& Siemann, E. (1997). The influence of functional diversity and composition on ecosystem $1300-1302$. https://doi.org/10.1126/science.277.5330.1300

Timm, R. M., LaVal, R. K., \& Rodríguez-Herrera, B. (1999). Clave de campo para los murciélagos de Costa Rica. Brenesia, 52, 1-32.

Vargas-Espinoza, A., Aguirre, L. F., Swarner, M., Emmons, L., \& Teran, M. (2004). Distribución de Vampyrum spectrum en Bolivia y comentarios sobre su estado de conservación. Ecología en Bolivia, 39(2), 46-51.

Villéger, S., Mason, N. W., \& Mouillot, D. (2008). New multidimensional functional diversity indices for a multifaceted framework in functional ecology. Ecology, 89(8), 2290-2301. https://doi.org/10.1890/07-1206.1

Villéger, S., Miranda, J. R., Hernández, D. F., \& Mouillot, D. (2010). Contrasting changes in taxonomic vs. functional diversity of tropical fish communities after habitat degradation. Ecological Applications, 20(6), 1512-1522. https://doi.org/10.1890/09-1310.1

Violle, C., Navas, M. L., Vile, D., Kazakou, E., Fortunel, C., Hummel, I., \& Garnier, E. (2007). Let the concept of trait be functional!. Oikos, 116(5), 882-892. https://doi.org/10.1111/j.0030-1299.2007.15559.x

Ward, J. H. (1963). Hierarchical grouping to optimize an objective function. Journal of the American Statistical Association, 58(301), 236-244. https://doi.org/10.1080/01621459.1963.10500845

York, H. A., Rodríguez-Herrera, B., LaVal, R. K., Timm, R. M., \& Lindsay, K. E. (2019). Field key to the bats of Costa Rica and Nicaragua. Journal of Mammalogy, 100(6), 1726-1749. https://doi.org/10.1093/jmammal/gyz150 